\title{
ABSORPTION COLLAPSE IN PRIMARY TUBERCULOUS INFECTION IN CHILDHOOD
}

\author{
BY \\ STANLEY GRAHAM, M.D., F.R.C.P.(Edin.), F.R.F.P.S.(Glas.) \\ and JAMES H. HUTCHISON, O.B.E., M.D., M.R.C.P.(Lond.), F.R.F.P.S.(Glas.)
}

(From the Department of Medical Paediatrics, University of Glasgow, and the Royal Hospital for Sick Children, Glasgow)

Childhood tuberculosis has been the subject of much study during the past two decades, and a flood of light has already been thrown on to the life history of the disease. At the present time the conception of the primary lung complex is generally accepted. The inhaled tubercle bacilli find their way via the bronchi and bronchioles to an area in the lung just below the pleural surface. Here there is set up an area of bronchopneumonia varying in size from a grain of rice to a walnut. Drainage from this primary focus takes place via the peribronchial lymphatics to the regional lymph glands, and the term 'mediastinal tuberculosis' was at one time used to designate this condition. Routine tuberculin skin tests and recording of temperatures such as were undertaken by Wallgren (1935) show that this initial infection is usually accompanied by some degree of fever and malaise attributed to allergy, although clinical and radiological examination does not often reveal anything abnormal. A high erythrocyte sedimentation rate and a positive tuberculin skin test in a young child, however, indicate the necessity for care and continued observation. A follow-up of such positive reactors has revealed a varied course. By far the greatest number proceed to healing with no untoward episodes. Certain phenomena occasionally appear which are regarded as due to allergy, namely, erythema nodosum and phlyctenular conjunctivitis. Pleurisy with effusion, it has also been suggested, belongs to this category, although positive evidence of this is lacking. It seems not unlikely that this condition may arise occasionally, in older subjects at least, from infection of the pleura by the tuberculous process. We shall not discuss this further. Bronchogenic spread producing tuberculous bronchopneumonia, especially in infants, and haematogenous spread producing miliary tuberculosis and tuberculous meningitis in older children, are well recognized and with rare exceptions end in death within a relatively short time.
In addition to the above there also occur in children passing through their primary tuberculous infection extensive radiological signs of mischief in the lungs in the absence of any marked degree of toxaemia. The clinical picture accompanying these findings, and the course of the condition, were outlined by Elfasberg and Neuland (1920), and to it they gave the name of epituberculosis. It has been suggested that the extensive shadow seen in the radiograph is produced by the toxins in the lung tissue adjacent to the primary focus, that is, that it is a perifocal reaction such as occurs in the vicinity of any inflammatory focus, e.g., a furuncle. This conception gained in popularity, and it appeared a plausible theory. Such patients usually progressed to recovery, however, and the critical test, namely, the histology of the affected lung, was seldom available. That the lesion is a tuberculous bronchopneumonia caused by tubercle bacilli of low virulence has also to be considered, but its frequency and good prognosis ${ }^{-}$would seem to be strong points against such a theory. Moreover, it does not behave as bronchopneumonia. The third suggestion is that such lesions can be explained by the pressure effects of the tuberculous regional lymph glands on the adjacent bronchi producing various degrees of occlusion, which in turn leads to collapse of the corresponding lobe or sector of a lobe (absorption collapse). This communication is presented with the object of providing evidence in favour of this latter theory.

\section{Clinical Manifestations}

The clinical material consists of a series of fortyfive cases admitted to the Royal Hospital for Sick Children, Glasgow, since 1939. Not all of the cases seen are included in the series, as some were not admitted for full investigation and others were not availáble for re-examination. In all cases the investigation included a family and personal history, a clinical examination, Mantoux and Pirquet tuberculin skin tests, estimation of the erythrocyte 
sedimentation rates, and radiography. In many the gastric residue was examined for tubercle bacilli by direct smear and by guinea-pig inoculation. In later cases lipiodol bronchography and bronchoscopy were used to determine the presence of the bronchial occlusion as well as its nature and degree.

A study of the clinical findings reveals that, although a diagnosis of tuberculous infection could be confirmed in every case by the presence of positive skin tests, the clinical signs were rarely such as to enable an accurate diagnosis of absorption collapse to be made, or for it to be possible to discover with any accuracy which lobe or sector was airless. In only nineteen cases were local signs detected clinically, such 'as impairment of the percussion note, . bronchial breath sounds, diminished air entry, or scanty râles, and these signs were never so marked as to justify more than a suspicion that absorption collapse had occurred, save in five cases where clinical evidence of mediastinal displacement confirmed the true cause of the signs. However, now that the true nature of these cases is recognized by us, the finding of minor localizing clinical signs in a child not acutely ill and with positive skin tests is regarded as indicating with little likelihood of error that further investigation will demonstrate the presence of pulmonary collapse. The diagnosis is thus made by a process of deduction from past experience and from the knowledge of the pathological course often pursued by primary tuberculosis, and is not based on the physical signs alone, which, as stated, do not by themselves justify any such clearly defined diagnosis. As experience and insight into this pulmonary complication has been acquired, we have come to regard another clinical sign seen in fifteen of our cases as being strongly suggestive evidence of pulmonary collapse, although its tuberculous nature of course depends on the presence of positive skin tests. We refer to those cases in which a history is obtained of asthma-like breathing, wheezy breathing, or spasms of nocturnal cough with respiratory distress; or, alternatively, in which auscultation reveals the presence of rhonchi over one lung without the other accompaniments of bronchitis or asthma. So characteristic, indeed, is this picture, that we now believe that the appearance of ' asthma ' in an infant under the age of two years, especially in the absence of a family history of allergy, should arouse a suspicion of tuberculous hilar adenitis with some degree of bronchial occlusion, and that this suspicion should be abandoned only after adequate investigation, including radiography and tuberculin skin testing. The significance of this syndrome of 'asthma', 'pertussis-like' cough, noisy breathing, wheezing, ' musical rhonchi ' in bronchial narrowing, has been recognized by previous authors (Jackson, 1930; Macpherson, 1939; Jones et al., 1942; Kent, 1942; Meneghello and Smith, 1943).

As regards the constitutional upset, it seems probable that the occurrence of collapse is associated with some fever and mild toxaemia in all or most cases, but in a few there was an acute initial illness with brisk fever, malaise, listlessness, anorexia, and infrequent dry cough. Loss of weight was the rule, but it was only marked in a few cases, especially in infants. In two of our forty-five cases there was concomitant erythema nodosum, and two others had pleurisy with effusion before the occurrence of the absorption collapse. It must be stressed that the occurrence of pulmonary collapse may often be attended by such minimal upset that neither the child nor his parents are in any way concerned by it. The history of some of our cases suggested that the collapse had existed for six months previously, during which time the patients' activities were little curtailed. Undoubtedly many children with a primary tuberculous infection complicated by pulmonary collapse pass through the complete illness unsuspected, and it is probably from this type of case that bronchial stenosis or uninfected upper lobe bronchiectasis discovered by routine examination arises.

For example, Lightwood and Wilson (1936) describe the case of a boy who three years previously had had an illness diagnosed as pneumonia. Five months before coming under their care he had another attack followed by a cough with a little sputum and some breathlessness, but no malaise or fever. The tuberculin skin tests were positive. Bronchial stenosis was demonstrated both by lipiodol bronchography and by bronchoscopy. The lung was removed and was found to be shrunken and fibrous (absorption collapse), and to contain numerous areas of calcification.

There is in our wards at the present time a somewhat similar case with bronchiectasis of the right middle lobe, where bronchoscopy and bronchography revealed a bronchial stenosis, the opening into the right mid-lobe bronchus being greatly constricted.

Source of infection. A history of contact with open pulmonary tuberculosis was obtained in seventeen of the forty-five cases. Contrary to expectation, the frequency of such a history was not greater in younger infants than in older children. Because of the smaller circle of contacts of the young child it seemed reasonable to suppose that the younger patients probably get infected from near relations or frequent household visitors.

Radiographic signs. Radiography was carried out in every case, and was frequently repeated over long periods in some. It was always possible with antero-posterior and lateral films to localize the area of collapse with fair accuracy. The incidence of lobes affected.was as follows:

$\begin{array}{lrrr}\text { Right upper lobe } \quad . & \ldots & \ldots & 10 \\ \text { Right middle lobe } \ldots & \ldots & \ldots & 13 \\ \text { Right upper and middle lobes } & \ldots & 1 \\ \text { Right lower lobe ... } \quad . . & . & 4 \\ \text { Right upper and lower lobes } & \ldots & 1 \\ \text { Right upper and left upper lobes } & \ldots & 1\end{array}$


$\begin{array}{lllll}\text { Left upper lobe } & \ldots & \ldots & \ldots & 7\end{array}$

Left lower lobe $\quad . . \quad \ldots \quad \ldots \quad \ldots 7$

Left upper and lower lobes $\quad \ldots \quad 1$

The right upper and/or middle lobes were affected in twenty-six cases (57.8 per cent.); the left upper and/or the left lower lobes were affected in sixteen cases (35.6 per cent.). The right lower lobe was the least likely to be affected. These figures agree closely with those of Jones et al. (1942), and have been explained on anatomical grounds by Brock et al. (1937), who point out that because of their anatomical relationships the left tracheo-bronchial group of glands are much more likely to obstruct the left main bronchus than are the right tracheobronchial group to interfere with the right bronchus. The explanation of the frequency of right upper and middle lobe collapse is that the broncho-pulmonary gland lying inferior to the eparterial bronchus in the angle between it and the right main bronchus is likely to distort and obstruct the entrances to the right upper or mid-lobe bronchi.

Radiographic appearances of pulmonary collapse are not difficult to recognize as a rule, although it is our experience that these shadows are frequently misinterpreted. This was no doubt a factor which lent powerful support to the view that they might be associated with allergy as a perifocal reaction. The antero-posterior and lateral appearances of collapse have been well described by Erwin (1939). Radiological evidence of mediastinal and/or tracheal shift to the affected side was found only in seventeen of our forty-five cases. Other classical signs of collapse, such as elevation of the diaphragm or 'crowding' of the ribs, were not seen in any case. These findings are in agreement with those of Kent (1942), and Jones et al. (1942), and the last-named authors point out that the comparative infrequency of these signs of collapse is to be attributed to the fact that when the bronchial lumen is occluded gradually (as in a slow process like tuberculous hilar adenitis), and especially if parenchymal tuberculosis is present in the affected lobe or sector, adjustments are made and the outpouring of fluid into the tissues may be more rapid than the absorption of air, so that the volume of the lobe may be as great as, or actually greater than, that of the normal lobe. Such a filled or 'drowned' lobe is not atelectatic in the true sense of ' imperfect expansion', although its aerating surface is as inadequate as an unexpanded lobe; thus the term 'absorption collapse' more accurately describes the condition of lung tissue distal to the seat of bronchial occlusion, whether its volume be greater or less than normal.

It was not possible to follow all the forty-five cases for long periods. Some cleared completely and a few showed extensive calcification not only of the hilar glands, but within the substance of the collapsed lobe. Whether this peripheral calcification represented healing of broncho-pulmonary glands, peribronchial lymphatics, or the primary lung focus itself, it is difficult to say. In some cases the collapse shifted from one lobe to another from time to time, and in others the extent of the shadow of collapse in one lobe varied in size and position from time to time. This irregular behaviour of collapse, due to gradual and often partial bronchial occlusion, e.g. from extrinsic pressure by enlarged glands, is not surprising. Thus at one time the narrowed bronchial lumen may become completely obstructed by sticky mucus which may later be expelled bycoughing or postural changes and permit partial re-opening of the lumen for a time. Again, if a main bronchus be narrowed and the mucous membrane swollen and inflamed, the secretions therefrom may occlude the entry to an upper-lobe bronchus at one time and the entry to a lowerlobe bronchus at another.

Obstructive emphysema. The presence of partial bronchial occlusion may also, on occasions, produce the opposite of absorption collapse. Thus, partial obstruction by extrinsic pressure associated with a swollen hyperaemic mucous membrane may allow the entry of air to the alveoli distal to the site of the bronchial narrowing during inspiration, when the bronchi normally dilate slightly, but during expiration the slight narrowing of the bronchus may result in complete obstruction at the narrowed part of the lumen so that the exit of air cannot take place. This causes ballooning of the air-spaces, known as obstructive emphysema. This mechanism was first described accurately by Chevalier Jackson (1930). The radiograph of obstructive emphysema shows an area of increased translucency with possibly displacement of the mediastinum to the opposite side. Obstructive emphysema was seen twice in this series (cases 4 and 6). In case 4, the emphysema might have been compensatory to right upper lobe collapse, but the bronchogram showed marked narrowing of the right main bronchus. In case 6 the affected lobe had previously been collapsed. Meneghello and Smith (1943) in a series of fifteen cases showing bronchial obstruction, described obstructive emphysema in seven.

Bronchiectasis. In four of our cases of absorption collapse, bronchiectasis developed while they were under observation and, although the bronchiectasis was extensive in every case, it was outstandingly free from signs of secondary infection; thus, none of these children had any appreciable amount of sputum, there was no cachexia, and the appetite and general well-being were good. It is our impression that although bronchiectasis may be a not uncommon sequel to tuberculous pulmonary collapse in childhood, it seems to remain uninfected at least for many months. We would agree with Kent (1942), who described seven cases of bronchiectasis out of a total of ten cases of tuberculous bronchial obstruction, that changes of this nature do not play an appreciable role in the etiology of infected bronchiectasis. Uninfected bronchiectasis, often of an upper lobe, is a clinical phenomenon known to every chest physician. It is tempting to suggest that such a case often arises from a tuberculous collapse occurring during childhood. 
Bronchography. Lipiodól bronchography entails certain disadvantages in tuberculous infection because the lipiodol tends to persist in small amounts for a long time afterwards and often makes interpretation of subsequent $x$-ray films difficult. Bronchography was performed in seven cases in the present series in an attempt to obtain further evidence that there was, in fact, bronchial obstruction. This was successfully proved. In three cases an upper-lobe bronchus was shown to be completely occluded just distal to its origin, and in one of these cases marked constriction of the main bronchus was also revealed (case 4). In two cases the main bronchus leading to a collapsed right middle lobe was shown to be narrowed, distorted, tortuous, and to fill irregularly and poorly with lipiodol. In one of these cases the lateral division of the right middle lobe bronchus was completely occluded; in the other, both lateral and medial divisions were abruptly occluded just distal to their bifurcation from the main middle lobe stem. In the remaining two cases the bronchogram was taken to confirm the presence of bronchiectasis suspected of being present because these children had suffered from collapse for many months. Cylindrical bronchiectasis was revealed in both cases, and in one (case 6) the main bronchus leading to the bronchiectatic lower lobe was seen to be much constricted in comparison with the bronchus on the other side.

Bronchoscopy. Bronchoscopy has only recently been employed, and five cases of this series have been so examined. In two, a main bronchus was much narrowed by extrinsic pressure. In another (case 5) extrinsic narrowing was associated with ulceration and granulation tissue in the bronchial lumen. In the fourth case, one of right mid-lobe collapse, the entry to the mid-lobe bronchus was much narrowed by swollen hyperaemic mucous membrane which bled easily and a smear from which revealed tubercle bacilli. In the fifth case, that of an infant (case 1), bronchoscopy failed because of narrowing and kinking of the trachea, possibly from paratracheal adenitis.

Four cases have come to necropsy. In none of these had bronchoscopy been carried out. In one, that of a three-month infant, the clinical diagnosis of collapse was confirmed (case 7). This child died of tuberculous bronchopneumonia. The three other children died of tuberculous meningitis at the ages of six months, eleven months, and two years respectively, but unfortunately the necropsy records are incomplete. Several case-histories of individual patients are detailed, illustrating various points already outlined.

\section{Case Reports}

CASE 1. A boy aged nine months was admitted to hospital on Aug. 29, 1946. He was a full-term healthy infant of healthy parents, breast-fed for eight months and given cod-liver oil and orangejuice regularly. He thrived until the first week in June, 1946, when he developed wheezy, difficult breathing which persisted until the time of admission. In the three weeks before admission the breathing had become ' panting,' with drawing-in of the chest wall. He had lost weight slowly from the age of six months. There was no cough.

On examination he appeared to have lost weight, and he was, in fact, only 74 per cent. of the expected weight for his age. There was no pyrexia, fingerclubbing, or cyanosis. Breathing was distinctly laboured, with marked recession of the chest wall at the level of the diaphragm with each inspiration. Local signs included rhonchi heard over both lungs with prolongation of expiration. The heart sounds were best heard to the right of the sternum; the apex beat was not palpable. The spleen was enlarged one finger's breadth below the costal margin. The Mantoux and Pirquet tuberculin skin tests were strongly positive. Films of the gastric residue revealed tubercle bacilli. The cerebrospinal fluid was normal. The radiograph showed collapse of the right upper lobe with mediastinal displacement of such a degree as to produce dextrocardia. Bronchoscopy was attempted, but failed because the trachea was markedly narrowed by a bulge on the posterior wall and kinked to the right by the enlarged paratracheal glands. He was discharged home to the care of his family doctor on September 8, 1946.

CASE 2. A girl, aged six years, was admitted to hospital on May 30,1946. There was no family history of tuberculosis. She was a full-term healthy infant, breast-fed for four months and then fed on cows' milk with irregular vitamin supplements. She throve and developed normally. She had an attack of uncomplicated measles at one year. One month before admission she lost her appetite and became pale, listless, and easily tired. She was ' feverish' at night. There was no cough.

On examination she appeared reasonably healthy and was 91 per cent. of the expected weight for her age. There was no fever, cyanosis, or clubbing. The Mantoux and Pirquet tuberculin tests were strongly positive. The percussion note was unchanged, but there was slightly defective air-entry over the right upper chest. The sedimentation rate was $65 \mathrm{~mm}$. in the first hour. Films of the gastric residue did not reveal any tubercle bacilli. The radiograph (Plate Ia) showed collapse of the right upper lobe. The lipiodol bronchogram on June 11, 1946, showed a complete block of the right upperlobe bronchus just distal to its origin (Plate Ib). She was dismissed to be nursed at home on June 22, 1946. The radiograph on July 18 , showed partial re-expansion of the right upper lobe and a new collapse of the right middle lobe.

CASE 3. A boy aged $12 \frac{1}{2}$ years was admitted to hospital on July 6,1946 . There was no family history of tuberculosis. He had been a full-term healthy infant fed on Nestle's milk. He had had measles at three years, pertussis at four years, and scarlet fever at nine years. He had been well until July, 1945, since when he had had frequent colds, 
had been very listless, and had lost weight steadily. There was no cough except during the 'colds.' He was unduly breathless on exertion.

On examination he was found to be a pale, thin child, 75 per cent. of the expected weight for his age. There was no fever, cyanosis, or clubbing. The Mantoux and Pirquet tuberculin skin tests were strongly positive. The blood sedimentation rate was $25 \mathrm{~mm}$. in the first hour. There was a small area of dullness on percussion to the left of the manubrium sterni, and the breath-sounds were harsh all over the lefi lung. The radiograph showed collapse of the left upper lobe with marked shrinkage, and emphysema of the left lower lobe. The lipiodol bronchogram on July 10, 1946, showed complete block of the left upper lobe bronchus just distal to its origin. Bronchoscopy on Aug. 5, 1946, showed that at the level of origin of the left upper bronchus there was a large bulge in the posterior wall of the left main bronchus reducing its lumen by two-thirds. The mucous membrane over this bulge was swollen and hyperaemic but appeared intact. Films of mucus from this area did not reveal tubercle bacilli. On Aug. 29 the radiograph showed complete re-expansion of the left upper lobe.

CASE 4. A girl aged six and a half years was first admitted to hospital on March 17, 1946. There was no family history of tuberculosis. She was a full-term healthy infant, fed on Ostermilk and given vitamin supplements. She had had measles at three years, chicken-pox at four years, and pertussis at four and a half years. In mid-Dec., 1945, she had an attack of abdominal pain with fever which subsided quickly. Three weeks before admission she developed a cough which has persisted. The cough is worse at night, associated with breathlessness, and followed by a spell of wheezy, noisy breathing. On March 14 she had an epileptic fit. She had been feverish for some days before admission and had lost much weight.

On examination she appeared pale and ill, but was 89 per cent. of the expected weight for her age. The cough was loose. There was no dyspnoea, cyanosis, or clubbing. The rectal temperature was $101^{\circ}$ to $102^{\circ} \mathrm{F}$. for some days after admission. The Mantoux and Pirquet tuberculin tests were strongly positive. There were abundant rhonchi over both lungs ' as in asthma.' The blood sedimentation rate was $60 \mathrm{~mm}$. in the first hour. Antero-posterior and lateral radiographs showed the appearances of collapse of the subapical and pectoral axillary segments of the right upper lobe (Brock, 1946). Owing to shortage of beds she was sent home on March 31, 1945, to complete rest in bed. On May 17 she was re-admitted from the out-patient department because the antero-posterior and right lateral radiographs (Plate Ic and d) showed the characteristic appearances of complete collapse of the right upper lobe, with the 'gull-wing' shadow of the lower border in the lateral view, and emphysema, presumably obstructive, of the right lower lobe. She did not appear more ill at this time, and she was afebrile. The blood sedimentation rate was $35 \mathrm{~mm}$. in the first hour. The sputum was negative for tubercle bacilli. The lipiodol bronchogram on May 20,1946, showed complete occlusion of the right upper-lobe bronchus (Plate IIe), and the lateral view showed marked narrowing of the right main bronchus with only a trickle of lipiodol passing through (Plate IIf). On June 9 she had another convulsion, and on June 17 she was dismissed home on phenobarbitone. A radiograph taken as an out patient on Aug. 13 showed re-expansion of the right upper lobe, which now had multiple bronchiectatic cavities. She seemed fairly well, and there was very little sputum. The mother allowed her to be up for part of the day, contrary to advice. She was re-admitted on Aug. 21, having had a high fever for three days with a frequent dry cough. She now appeared flushed, dyspnoeic, and acutely ill. The temperature was $102^{\circ}$ to $104^{\circ} \mathrm{F}$. and this persisted thereafter in the ward. The blood sedimentation rate was $30 \mathrm{~mm}$. in the first hour, and the gastric residue contained numerous tubercle bacilli. Clinically the trachea was noted to be displaced to the right. The percussion note was slightly impaired over the right chest, but the breath-sounds were almost inaudible over all of the right lung, suggesting complete occlusion of the right main bronchus. Radiographs on Aug. 22, however, showed bronchiectasis in the right upper lobe and collapse of the right middle lobe. Because of the continued high fever she was given sulphamezathine, $0.5 \mathrm{~g}$. four-hourly, from Aug. 23, and penicillin, 15,000 units intramuscularly threehourly, from July 25 to Aug. 31, without any beneficial effect. Blood-culture, stool-culture and Widal reaction were negative. The leucocytes varied from 3,400 to 4,000 per c.mm. On Sept. 5 the dullness over the right back became marked, with distant amphoric breath-sounds. Bloodstained fluid was aspirated from the right pleural cavity. The protein content of this fluid was 4 per cent.; and the leucocytes were 75 per cent. lymphocytes, 20 per cent. polymorphonuclear cells, and 5 per cent. endothelial cells. At the time of writing she was going down hill rapidly, with continuous fever, cachexia, finger-clubbing, and frequent unproductive cough. The condition appeared to be a terminal tuberculous bronchopneumonia, which in our experience is a rare sequel to collapse at this age.

CASE 5. A boy aged six and a half years was first admitted to hospital on May 15, 1946. He had recently lived in the same house with an adult cousin later diagnosed as suffering from phthisis. He was a full-term six pound twin, healthy at birth, and fed on cows' milk. At the age of three weeks he was successfully treated for pyloric stenosis. He had whooping-cough at fourteen months, and measles in April, 1946. During the measles he had had an attack of severe abdominal pain and had not been well since, complaining of listlessness, 
anorexia, and loss of weight. On May 15 he was seen by his doctor because of severe right-sided abdominal pain, fever, and vomiting, for which he was admitted to the surgical wards.

On May 18 he was transferred to the medical wards still complaining of abdominal colic, and with fever which continued for another eight days. There were no signs of intrathoracic disease. The Mantoux and Pirquet tuberculin tests were positive and the blood sedimentation rate was $45 \mathrm{~mm}$. in the first hour. Because of these findings a radiograph was taken on May 20 which showed enlarged hilar and right paratracheal glands with a small opacity in the right upper lobe thought to be a primary focus. On June 3, when he was free from symptoms and afebrile, the radiographs showed a triangular shadow situated in the right upper lobe with its base at the right hilum, and the appearance of collapse of the lung sector supplied by the pectoral branch of the right upper lobe bronchus. At this time there was an impaired note in the right axilla. He was dismissed home on June 11. At the out-patient clinic on June 27,1946 , a radiograph revealed an extension of the area of collapse in the right upper lobe. (His twin was examined at this time, and the radiograph of his chest showed an area of collapse identical with that of his brother.) The patient was re-admitted to hospital on Aug. 15, 1946. The blood sedimentation rate was now $13 \mathrm{~mm}$. in the first hour. No tubercle bacilli could be demonstrated in the gastric residue, and guinea-pig inoculation was also negative. On Aug. 27 the condition was unchanged in the radiograph, so on Sept. 1 bronchoscopy was carried out. There was marked narrowing of the lumen of the right main bronchus from extrinsic pressure. On the medial aspect of this bronchus was an area of ulceration, covered with a thin layer of granulation tissue which could not be grasped with the crocodile forceps. The opening of the right upper bronchus was' almost obliterated by swelling of the minor carina.

CASE 6. A girl aged five and a half years was under observation in the out-patient department for three months. There was a history of past contact with two aunts and an uncle, all victims of open phthisis. She had been a full-term healthy infant, breast-fed for nine months. She had had whoopingcough in 1941, measles in 1942, and chicken-pox in 1943. In December, 1944, she had pneumonia, and since then had never really been well. She complained of tiredness, listlessness and lack of appetite. Three months before admission these symptoms became worse, with loss of weight and a loose frequent cough. The Mantoux and Pirquet tuberculin tests were positive. Radiographs (Plate IIg and $h$ ) on March 5, 1946, showed collapse of the left lower lobe with mediastinal displacement to the left, and the film taken on April 30 showed obstructive emphysema in the left lower zone, the heart having returned to the normal position (Plate IIIj). On May 16 there was a reappearance of the collapse of the left lower lobe and mediastinal displacement, and on June 4 obstructive emphysema was once again noticed; this time it affected the whole left lung, with the heart not displaced.

On admission to hospital on June 14, 1946, she was found to be 110 per cent. of the expected weight for her age. There was no clubbing or cyanosis. The Mantoux and Pirquet tuberculin tests were positive. The sputum was negative for tubercle bacilli on two occasions and by guinea-pig inoculation. The percussion note was dull at the left base, where after postural drainage a small area of bronchial breathing was discovered. Scanty râles were heard over the left base behind. A radiograph on June 25 showed collapse of the left lower lobe with the heart displaced to the left. The lipiodol bronchogram on June 28 (Plate IIIk) showed cylindrical bronchiectasis of the left lower lobe with marked constriction of the left main bronchus. At this time the child was in good condition, eating well, and coughing up only two drachms of sputum per day. She was discharged to a convalescent home on Aug. 5, 1946.

CASE 7. A girl aged three months was admitted to hospital on March 10, 1946, as an emergency. No history of contact with tuberculosis could' be obtained at the time, although it was later discovered that the father had phthisis. She had weighed $5.5 \mathrm{lb}$. at birth, and had been healthy and breast fed until three weeks before admission, when the feed was changed to Sister Laura's Food. She had had a cough since three days old, but on Feb. 17, 1946 , it became worse and the breathing was distressed, the condition being uninfluenced by the administrsttion of sulphadiazine.

On examination she was found to be a desperately ill, marasmic infant, 65 per cent. of the expected weight for her age. She was cyanosed and dyspnoeic and had a loose, frequent cough. The Mantoux and Pirquet tuberculin tests were strongly positive. Sputum obtained from the back of the throat contained abundant tubercle bacilli. The percussion note over the lungs was resonant throughout, but the breath-sounds were diminished at the right base. Râles were heard over the right chest. The temperature ranged from $101^{\circ}$ to $103^{\circ} \mathrm{F}$. until death on March 16, 1946. . The radiograph taken on March 13 showed the triangular shadow of collapse of the right lower lobe, with marked displacement of the mediastinum to the right. There was a diffuse mottling in the left upper zone.

Necropsy showed a generalized miliary tuberculosis. There was complete collapse of the right lower and middle lobes and the pectoral segment of the right upper lobe. Primary foci were found in the right lower and left upper lobes, both being surrounded by areas of caseous pneumonia. The right lower lobe bronchus was surrounded by a caseous broncho-pulmonary gland. Proximal to this the right lower and middle lobe bronchi were completely occluded by tenacious mucus. The bronchial occlusion in this case seemed to be due 
to a combination of narrowing from extrinsic glandular pressure and obstruction from thick mucus produced by a hyperaemic mucous membrane.

\section{Discussion}

The suggestion that pulmonary collapse may be brought about by occlusion of bronchi by pressure of glands is not new. In 1850, Gairdner, writing ' On the Pathological States of the Lung Connected with Bronchitis and Bronchial Obstruction,' cites a case which is similar to those under discussion and which is so complete that it is deemed worthy of quoting. A child of five years of age died of tuberculous meningitis. At necropsy the primary focus, which was the size of a walnut, was found in the upper margin of the left lower lobe. The enlarged glands at the root of the left lung pressed upon some of the bronchi going to the left lower lobe, causing collapse of the anterior extremity of this lobe. In his article Gairdner says, 'I think the frequency of collapse of the pulmonary tissue both in the adult and in the child must now be considered as established and its connexion with bronchial obstruction rendered at least extremely probable.' He quotes several authors who had arrived at a similar conclusion (Louis, 1829; Alderson, 1831). Gairdner also quotes Carswell, who 'figured the case of a monkey in which the left bronchus was much compressed or rather obliterated by a mass of tuberculous glands.' The corresponding lung, he goes on to say, had diminished to less than a third of its normal bulk, while the opposite lung, of which the bronchus was free, presented emphysema in several places. At about this time, too, Mendelsohn and Traube had produced collapse experimentally in animals by occluding bronchi with lead shot and balls of paper.

This work apparently attracted little attention or was forgotten, and it is only within recent years that the importance of bronchial occlusion in the etiology of bronchiectasis and as an explanation of the collapse in the primary tuberculous infection has been recognized. Morlock and Pinchin (1933) were the first to describe bronchial obstruction due to tuberculosis which had been observed bronchoscopically. The subject was a boy of fourteen years in whom epituberculosis had been diagnosed. He had an 'asthmatoid wheeze' produced by right upper lobe collapse. On bronchoscopy, tuberculous granulation tissue bulging into the lumen of the bronchus and covered with normal mucous membrane was observed. After removal of the granulation tissue the radiograph cleared in four days. Similar cases have been described by Brock et al. (1937), Jones et al. (1942), and Kent (1942) among others. The alternative explanation of the etiology of the type of case under discussion, namely, epituberculosis, has been based on the papers published by Eliasberg and Neuland $(1920,1921)$. They regarded the dense shadows as being caused by non-specific pneumonia in a tuberculous subject, but Reichle (1933) suggested from the histology that the lesion was a tuberculous pneumonia without caseation. The popular conception of epituberculosis, however, is that the shadow, which appears either suddenly or slowly and then recedes gradually, is an allergic response around the primary focus. This is a very plausible theory, but, so far as we know, such a condition has not been described histologically in man, although Oppenheimer (1935) has produced a condition experimentally in animals which would seem to support the view that such an allergic response in lung tissue may occur. She produced a radiographic lesion like that of epituberculosis by the injection of dead tubercle bacilli into sensitized rabbits; no similar shadows appeared in the nonsensitized rabbits. Histologically the lesion is described as a 'typical tuberculous pneumonia,' but the course was one of regression and disappearance, leaving the animal unaffected.

However, all workers, including ourselves, who have set out to determine the true nature of the lesion in these cases have discovered evidence only of bronchial occlusion and collapse-by radiography, by bronchoscopy, and by pathological examination. It seems reasonable to conclude that, although primary tuberculous infection does produce spontaneous manifestations of allergy, such as erythema nodosum and phlyctenular conjunctivitis, epituberculosis is not one of these manifestations. Rather does its explanation rest purely on a wellfounded and easily demonstrated mechanical basis.

It might be suggested that, in a child in whom a bronchus is occluded by a mass of tuberculous granulation tissue containing virulent tubercle bacilli, bronchoscopy is contraindicated, and that the removal of the tuberculous granulation tissue via the bronchoscope might be followed by a rapid spread of the infection and the death of the patient. However, such does not seem to be the case (Morlock and Pinchin, 1933; Brock et al., 1937; Jones et al., 1942; Kent, 1942; Meneghello and Smith, 1943). On the other hand, failure to bring about expansion of the collapsed lung may result in the development of bronchiectasis. Further observations on the final results obtained by bronchoscopy and removal of the obstruction compared with those not so treated are urgently required.

\section{Summary}

Absorption collapse of a lobe or sector of a lobe due to bronchial occlusion is of common occurrence 
in the course of the primary tuberculous infection in children. In some cases of incomplete bronchial occlusion, obstructive emphysema may result.

The term 'epituberculosis' as applied to this condition is a misnomer, and the suggestion that the shadow seen in the radiograph indicates a perifocal reaction of an allergic nature is not justified.

\section{REFERENCES}

Brock, R. C. (1946). The Anatomy of the Bronchial Tree. London.

—, Cann, R. J., and Dickinson, J. R. (1937). Guy's Hosp. Rep., 87, 295.

Eliasberg, H., and Neuland, W. (1920). Jahrb. Kinderheilk., 93, 88. (1921). Ibid., 94, 102.

Erwin, G. S. (1939). Brompton Hosp. Rep., 8, 43.
Gairdner, W. T. (1850). Monthly J. med. Sci., 11, 122.

Jackson, C. (1930). J. Amer. med. Ass., 95, 639.

Jones, E. M., Rafferty, T. N., and Willis, H. S. (1942). Amer. Rev. Tuberc., 46, 392.

Kent, E. M. (1942). Ibid., 46, 524.

Lightwood, R., and Wilson, R. (1936). Arch. Dis. Childh., 11, 321.

Macpherson, A. M. C. (1939). Brompton Hosp. Rep., 8, 30 .

Meneghello, J., and Smith, C. A. (1943). J. Pediat., 22, 265.

Morlock, H. V., and Pinchin, A. J. S. (1933). Lancet, 1, 1114.

Oppenheimer, E. H. (1935). Johns Hopk. Hosp. Bull., 57, 247.

Reichle, H. S. (1933). Amer. J. Dis. Child., 45, 307.

Wallgren, A. (1935). Ibid., 49, 1105.

(For illustrations to this article see Plates I-III.) 


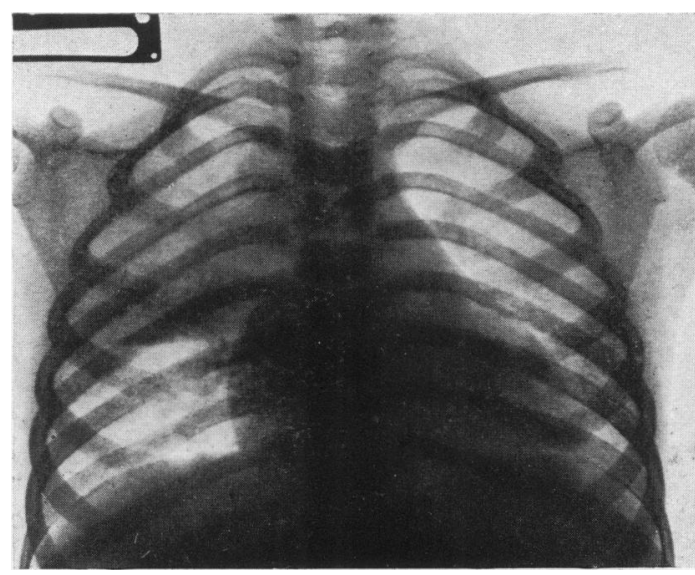

(a).-Showing collapse of right upper lobe (case 2).

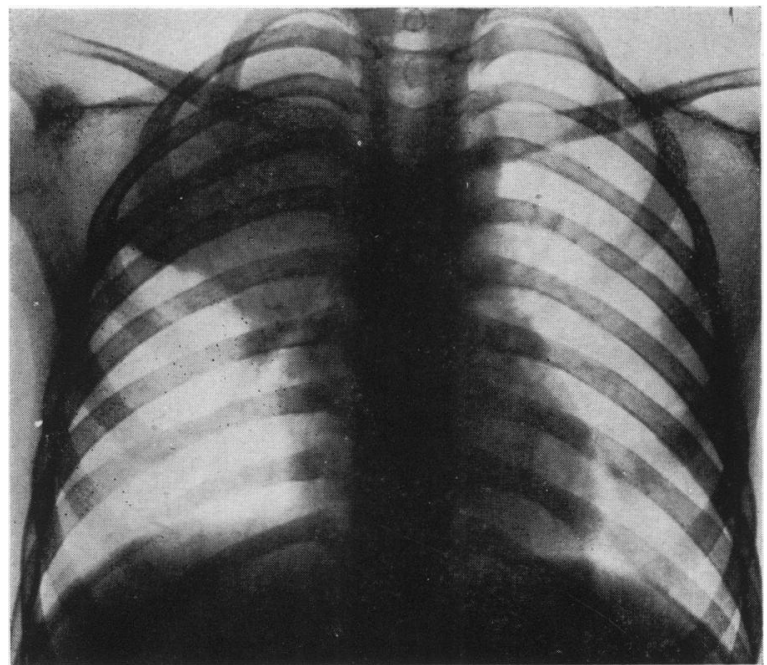

(c).-Antero-posterior view showing collapse of right upper lobe and obstructive emphysema of right lower lobe (case 4).

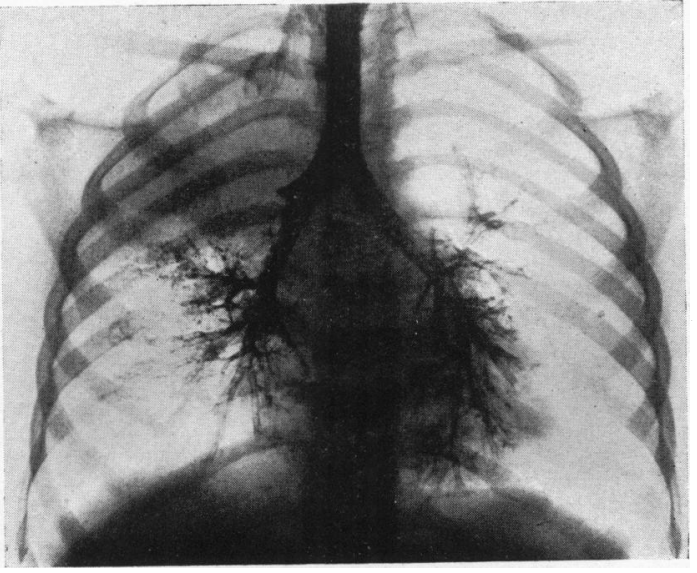

(b).- Showing block of right upper-lobe bronchus (case 2).

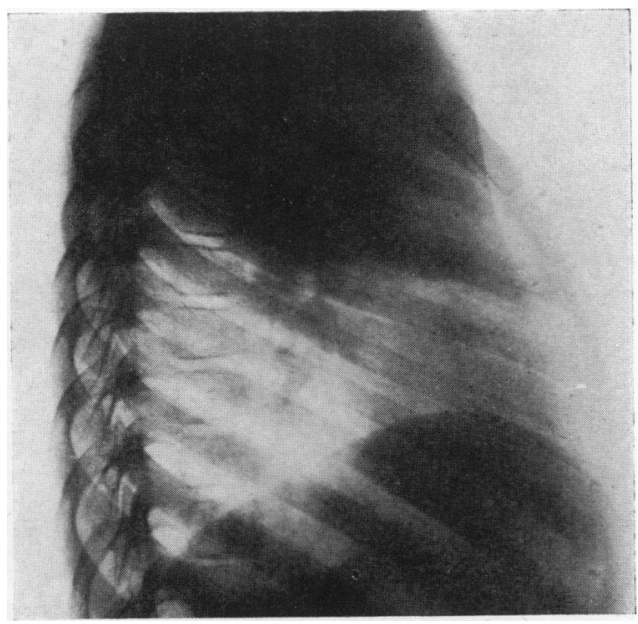

(d).-Right lateral view showing ' gull-wing' lower border of completely collapsed right upper lobe (case 4). 


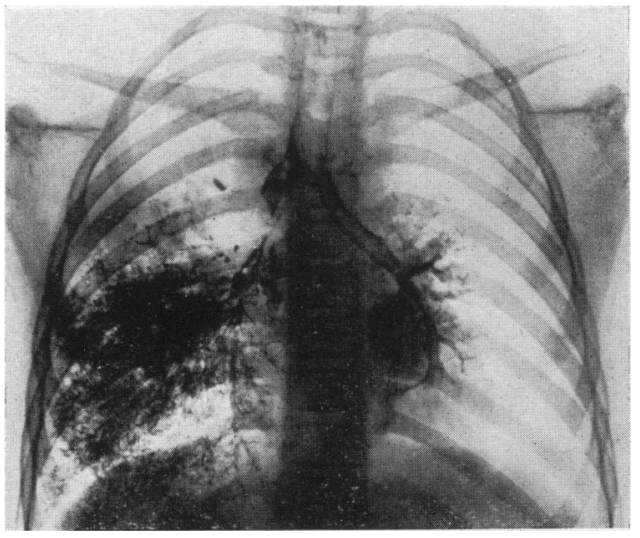

(e).- Showing complete occlusion of right upperlobe bronchus (case 4).

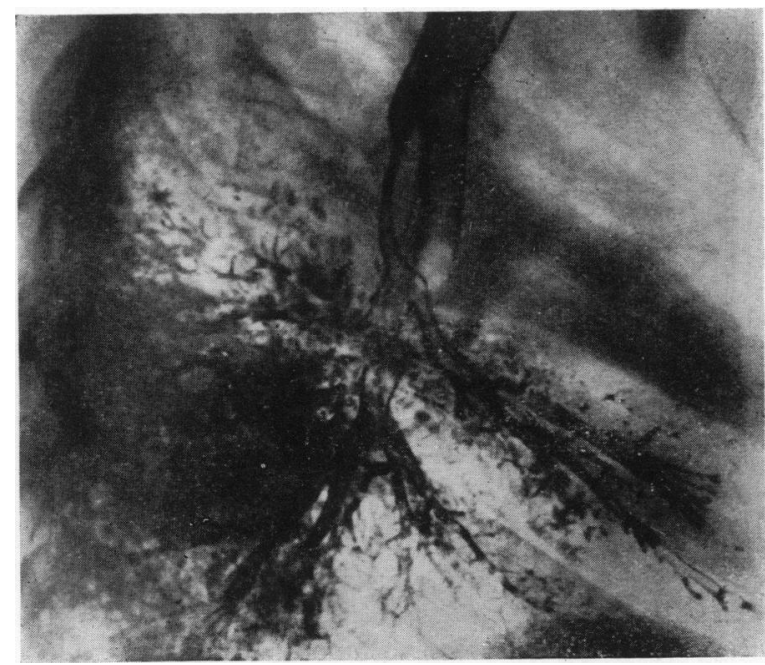

(f).--Right lateral view showing marked constriction of the right main bronchus (case 4).

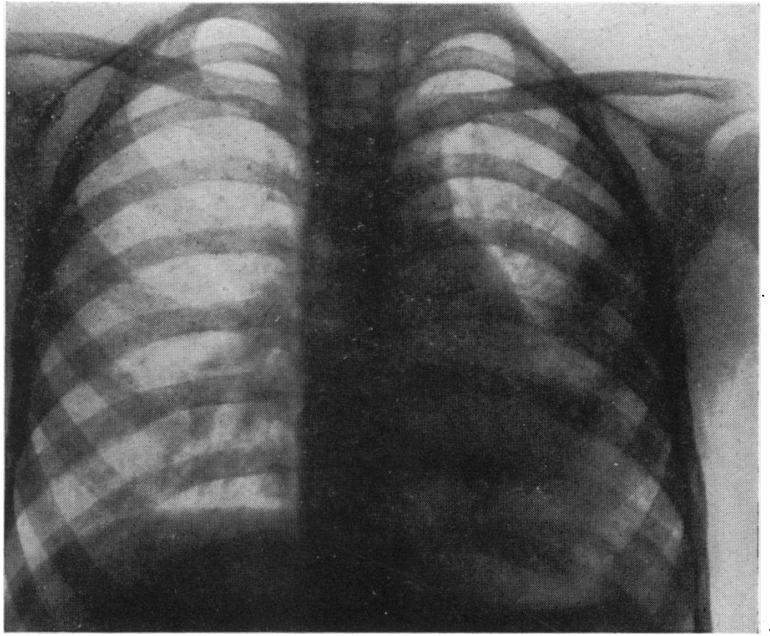

(g).-Antero-posterior view showing collapse of left lower lobe with mediastinal shift to the left (case 6).

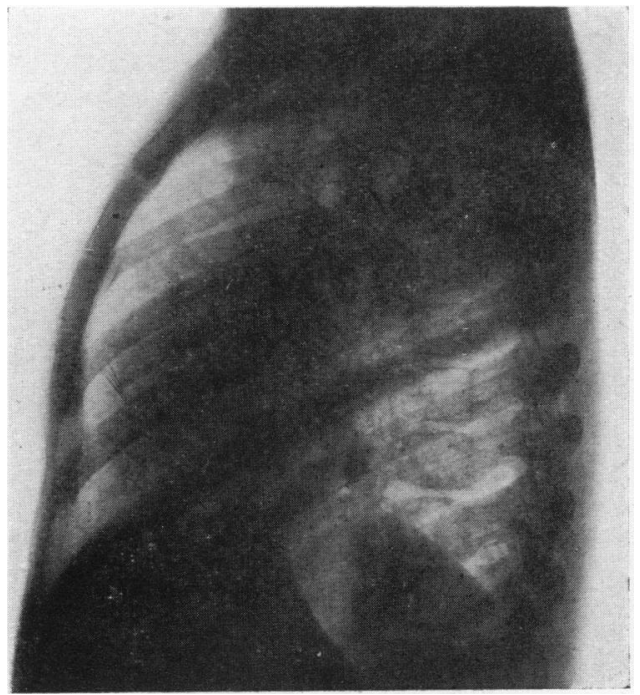

(h).-Left lateral view showing collapse of the left lower lobe (case 6). 


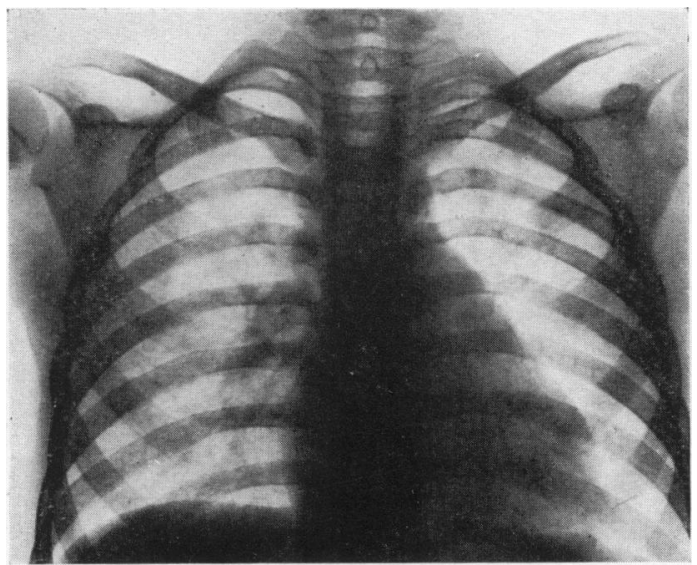

(j).--Antero-posterior view showing obstructive emphysema of the left lower lobe, with return of mediastinium to the normal position (case 6).

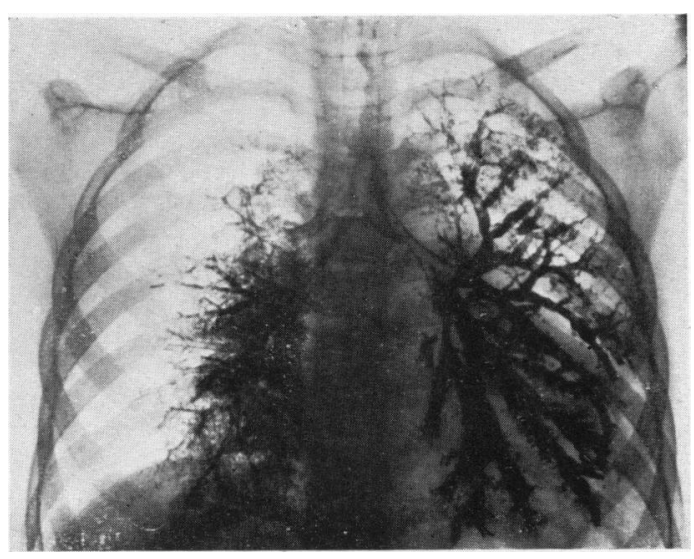

(k).- Showing cylindrical bronchiectasis of the left lower lobe, with narrowing of the left main bronchus (case 6).

\section{OSTEOMYELITIS OF THE MAXILLA IN INFANCY BY J. C. HAWORTH}

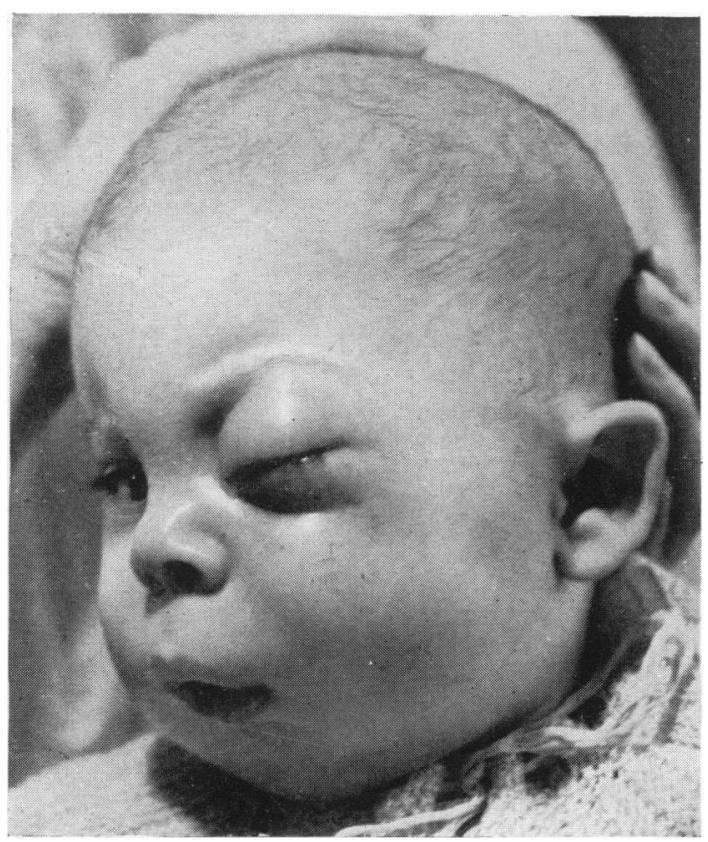

(a)-Case 4. Condition on admission to hospital.

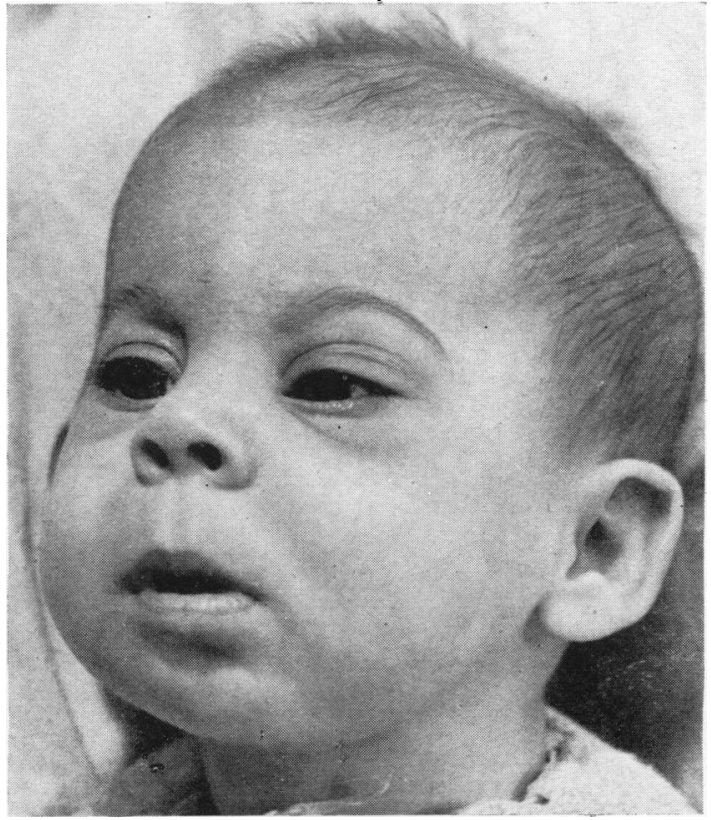

(b)-Case 4. Condition on discharge from hospital. 